\title{
SPARCL1, a Novel Prognostic Predictive Factor for GI Malignancies: a Meta-Analysis
}

\author{
Hanguang Hu $\mathrm{H}^{\mathrm{a}, \mathrm{b}}$ Wen Cai ${ }^{\mathrm{a}, \mathrm{b}} \quad$ Shu Zheng ${ }^{\mathrm{b}}$ Weiting Ge ${ }^{\mathrm{b}}$ \\ aDepartment of Medical Oncology, the Second Affiliated Hospital, School of Medicine, Zhejiang \\ University, Hangzhou, Zhejiang, ${ }^{b}$ Cancer Institute (Key Laboratory of Cancer Prevention and \\ Intervention, China National Ministry of Education), the Second Affiliated Hospital, School of Medicine, \\ Zhejiang University, Hangzhou, Zhejiang, China
}

\section{Key Words}

SPARCL1 • Meta-analysis • Gastro-Intestinal (GI) malignancies • Prognostic prediction factor

\begin{abstract}
:
Background/Aims: Secreted protein acidic and rich in cysteines-like 1 (SPARCL1) is abnormally expressed in gastrointestinal (GI) malignancies. However, the correlation between SPARCL1 expression and the prognosis of patients remains unknown. Therefore, we performed a metaanalysis to investigate the potential value of SPARCL1 as a prognostic predictive marker for GI malignancies. Methods: The PubMed, Embase, EBSCO, CNKI, and Wanfang databases were systematically searched for studies examining SPARCL1 and clinicopathological features, including the prognoses of patients. Hazard ratios (HRs) and odds ratios (ORs) from individual studies were calculated and pooled using a random-effects or fix-effects model. Heterogeneity and publication bias analyses were performed. Results: Data from 8 studies, including a total of 2,356 patients, were summarized. The expression of SPARCL1 suggested a better prognosis ( $\mathrm{HR}=0.57,95 \% \mathrm{CI}: 0.445-0.698, P=0.000)$ and was associated with clinicopathological features of GI malignancies, including distant metastasis ( $\mathrm{OR}=0.44$, 95\% CI: $0.23-0.85, P=0.014$ ), lymph node metastasis ( $\mathrm{OR}=0.56,95 \% \mathrm{CI}: 0.39-0.81, P=0.002)$ and tumor differentiation $(\mathrm{OR}=2.21$, 95\% CI: 1.82-2.69, $P=0.000$ ). Subgroup analyses based on cancer type revealed that the expression of SPARCL1 had no effect on lymph node metastasis in colorectal cancer, and it did not influence tumor differentiation in gastric cancer. Egger's test showed no evidence of publication bias (all $P>0.05$ ). Conclusion: SPARCL1 could be a novel prognostic predictive factor for GI malignancies. The expression of SPARCL1 could influence the clinicopathological features of GI malignancies. Further large-scale studies are essential to confirm SPARCL1's prognostic predictive value, and more fundamental experimental studies are needed to illustrate the mechanisms.
\end{abstract}

H. Hu and W. Cai share the first authorship.

Weiting Ge

KARGER
Cancer Institute, Key Laboratory of Cancer Prevention and Intervention, China National Ministry of Education, the Second Affiliated Hospital, School of Medicine, Zhejiang University, Hangzhou, Zhejiang (China); E-Mail geweiting@zju.edu.cn 


\section{Cellular Physiology Cell Physiol Biochem 2017;44:1485-1496 \begin{tabular}{l|l|l} 
and Biochemistry Published online: December 01, 2017 & $\begin{array}{l}\text { (c) } 2017 \text { The Author(s). Published by S. Karger AG, Basel } \\
\text { www.karger.com/cpb }\end{array}$
\end{tabular}}

Hanguang et al.: SPARCL1 as a Prognostic Marker for GI Malignances

\section{Introduction}

Secreted protein acidic and rich in cysteines-like 1 (SPARCL1) is one of the ten members of the SPARC-related family. As a secreted extracellular matrix glycoprotein, SPARCL1 was first discovered in 1995 and cloned from high endothelial venule (HEV) endothelial cells in human tonsil lymphatic tissue [1]. SPARCL1 expressed in normal tissues and organs such as gastrointestinal tract, heart, lung and brain, but it had a lower expression in pancreas, spleen, thyroid gland and placental tissue and not expressed in liver and peripheral blood leucocytes [2]. It participated in many physiological functions both during embryogenesis and in adult life during tissue remodeling.

Gastrointestinal (GI) malignancies are a group of cancers that affect the digestive system. These malignancies include cancers of the esophagus, biliary system, liver, pancreas, stomach, small intestine, bowel (large intestine or colon and rectum), and anus. GI malignancies such as colorectal cancer and gastric cancer are associated with high morbidity and mortality [3]. Furthermore, the burden of GI cancer is increasing, especially in Asia [4]. Therefore, identifying prognostic biomarkers will provide evidence for patients to choose the appropriate therapy.

The SPARCL1 gene was found to be downregulated in various GI malignancies [5, 6]. The SPARCL1 protein has been reported to possess multiple functions related to the processes of GI malignancy progression such as the regulation of cell adhesion, migration, and proliferation [7-9]. Therefore, SPARCL1 has been speculated to be an endothelial tumor marker associated with tumor progression.

However, to date, no pooled analyses on SPARCL1 have evaluated its value for predicting the prognosis of GI malignancies. Thus, this meta-analysis was conducted and included studies that assessed the role of SPARCL1 in the clinical outcomes of patients with GI malignancies.

\section{Materials and Methods}

\section{Literature search strategy}

All articles published were systematically searched using the PubMed, Embase, EBSCO, CNKI, and Wanfang databases without any language restrictions. The overall search strategy included the following search terms: "SPARCL1", "SPL1", “SC1", “MAST9”, “RAGS-1", “QR1", “ECM2", "Hevin” AND "tumor", "tumour”, "neoplasm", "cancer", "carcinoma”. Two investigators inspected the titles and abstracts and obtained the full texts. The investigators then selected only publications related to gastrointestinal malignancies. References from the relevant published review articles were manually searched to identify other possible studies. Conference abstracts or case reports were excluded due to the lack of sufficient data for meta-analysis.

\section{Inclusion and exclusion criteria}

Studies were eligible if they met the following initial inclusion criteria: (a) detected SPARCL1 in tumor tissue or serum; (b) clearly described the detection method of SPARCL1; (c) clearly defined the cut-off values of SPARCL1; (d) assessed the relationships between SPARCL1 expression and the clinicopathological features or prognosis of GI malignancies; and (e) correlated survival outcomes with SPARCL1 expression and provided sufficient data to estimate the odds ratio (OR) and hazard ratio (HR). Studies were excluded if they (a) were review articles, case reports, experimental studies, conference abstracts or letters; (b) included unpublished data; or (c) lacked essential data for the pooled calculation.

\section{Data extraction}

Eligible articles were reviewed independently by two reviewers (W.G and H.H). Disagreements were resolved by consensus and consultation with a third investigator (S.Z). Data were extracted independently by two authors (W.G and H.H) using a previously determined protocol. The following required information was collected: first author; year of publication; affiliated country; number of patients; tumor type; sample source; follow-up period; methods of detecting SPARCL1 expression; antibody used to detect SPARCL1; 


\section{Cellular Physiology Cell Physiol Biochem 2017;44:1485-1496 \begin{tabular}{l|l|l} 
and Biochemistry Published online: December 01, 2017 & $\begin{array}{l}\text { (c) } 2017 \text { The Author(s). Published by S. Karger AG, Basel } \\
\text { www.karger.com/cpb }\end{array}$
\end{tabular}}

definition of SPARCL1 cut-off value; lymph node status; differentiation of tumors and HR for survival outcomes as well as their $95 \%$ confidence interval (CI) and $P$-values.

\section{Statistical analyses}

Statistical analyses were performed using STATA version 12.0 software (Stata Corporation, Collage Station, Texas, USA). The expression of SPARCL1 was divided into an overexpression group and a positive group according to the described publications. The grade of tumor differentiation was divided into high (including well and moderately differentiated) and low (poorly differentiated) groups. Lymph node metastases were defined as having a lymph node-positive status. When the pooled HR was calculated, the multivariate Cox analysis data were included if available [10]. The pooled OR and corresponding 95\% CI were calculated to determine the correlation of SPARCL1 expression with distant metastasis, lymph node metastasis or tumor differentiation. Rounding was avoided when performing these calculations. Heterogeneity across studies was assessed using the Chi-square-based Q statistical test [11]. Subgroup analysis, sensitivity analysis and meta-regression were performed to determine the source of heterogeneity. Inter-study heterogeneity was assessed by visual inspection of the forest plots. A random-effects model was used when heterogeneity was present $\left(P<0.05\right.$ and/or $\left.\mathrm{I}^{2}>50 \%\right)$; otherwise, a fixed-effects model (Mantel-Haenszel method) was applied $[12,13]$. The publication bias was evaluated using funnel plots, Egger's test and Begg's test.

\section{Results}

\section{Description of studies}

A total of 102 studies were identified from a search of the above databases. We selected qualified publications using the strategy described in Fig. 1. After reviewing the abstracts or full-text of these studies, a total of 8 eligible studies were chosen for this meta-analysis [1421]. The features of the 8 included studies are summarized in Table 1 . These studies were published from 2011 to 2015 , and a total of 2356 patient samples were included to investigate the potential prognostic value of SPARCL1 expression and the relationship between the expression of SPARCL1 and the pathological features of the tumors. The clinical features of included patients are summarized in Table 2.Three types of GI malignances were included in the studies, namely, colorectal cancer, gastric cancer and biliary cancer. Sample sizes ranged from 48 to 1072 patients. Only 2 studies enrolled less than 100 patients. Five studies focused on overexpression of SPARCL1 (scoring), and 3 studies selected negative staining as the cut-off point. Tissue samples were used for detection of SPARCL1 expression. Goat polyclonal anti-human SPARCL1 IgG antibody (R\&D Systems, Minnesota. USA) was used for immune-histochemical staining. Six studies reported multivariate Cox analysis results for the relationship between patient prognostic prediction and SPARCL1 expression. Six studies that included a total of 261 patient samples with distant metastasis and 1989 patient samples without metastasis evaluated the relationship between SPARCL1 expression and distant metastasis. Six studies that included a total of 1187 lymph node-positive patients and 869 lymph node-negative patients assessed the correlation between SPARCL1 expression and lymph node status. Seven studies that included a total of 1450 well- or moderately-

Fig. 1. Flow chart of the search strategy and selected studies.

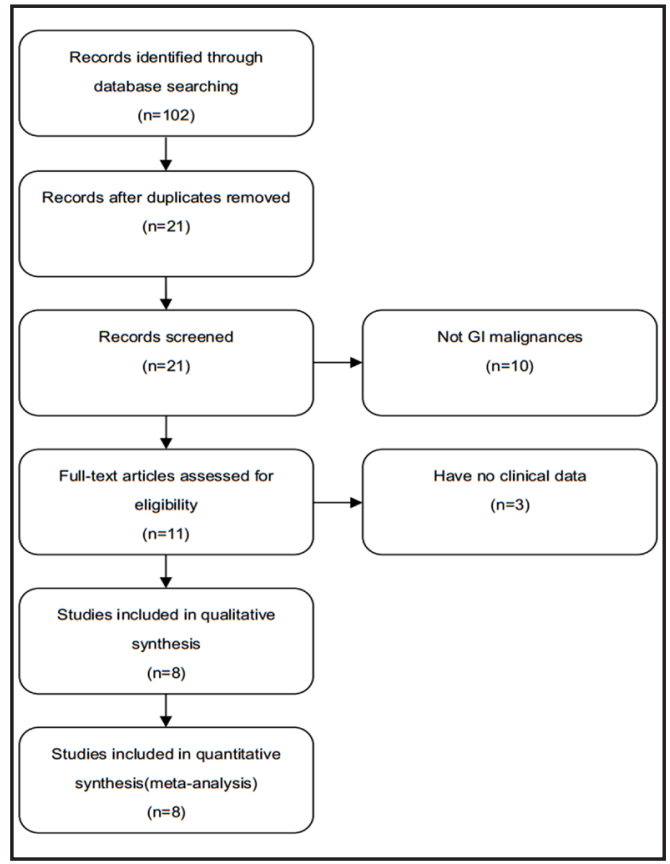


differentiated samples and 739 poorly-differentiated samples assessed the association of the expression of SPARCL1 with the tumor differentiation grade.

\section{Quality assessment}

Each of the 8 eligible studies included in our meta-analysis was assessed for quality according to the Newcastle-Ottawa Scale (NOS) [22]. The score of all included studies varied from 6 to 8, with a mean of 6.6. A higher value indicated better methodology. Therefore, all studies were included in the subsequent analysis.

\section{Meta-analysis results SPARCL1 could be a novel prognostic pre- dictive factor for GI malignancies}

This meta-analysis was performed on 6 studies. The pooled HR was $0.57 \quad(95 \%$ CI: $0.445-$ 0.698, $\mathrm{Z}=8.87, P=0.000$ ) with heterogeneity $\left(\mathrm{I}^{2}=0.0 \%, \quad P=0.666\right)$ using a fixed-effects model. These results suggested that the expression of SPARCL1 was a significantly valuable prognostic factor for a better prognosis of GI malignances. The subgroup analysis indicated that SPARCL1 was significantly associated with better survival among colorectal, gastric and biliary tract cancer patients $(\mathrm{HR}=0.54$, $\mathrm{HR}=0.62$, HR=0.52, respectively). The heterogeneity across the subgroups was not signifi-

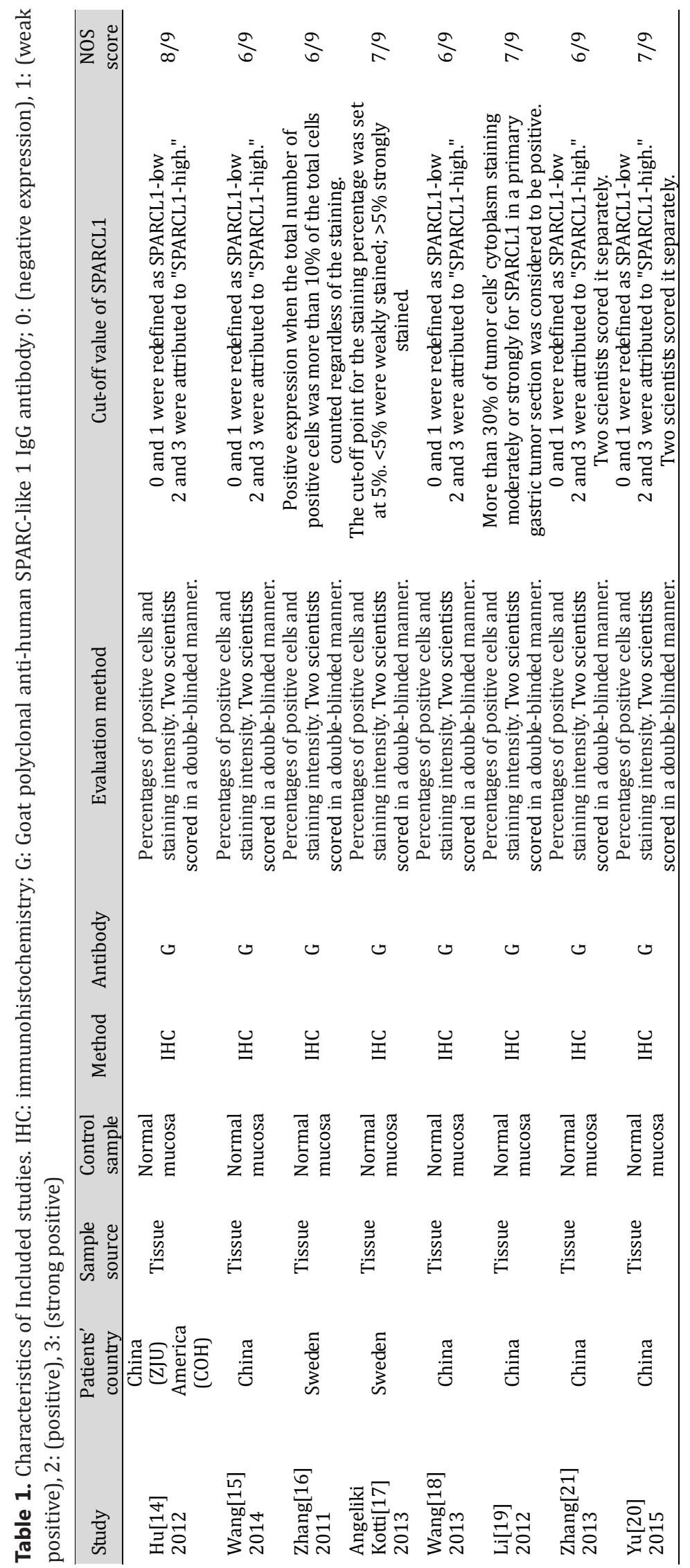


cantly different $(P=0.805$, Fig. 2).

The expression of SPARCL1 may suggest a better prognosis for tumor metastasis, lymph node status and tumor differentiation

Six studies evaluated the relationship between SPARCL1 expression and distant metastasis. The pooled OR was $0.59(95 \%$ CI: $\quad 0.33-1.06, \quad \mathrm{Z}=1.77$, $P=0.076)$ with heterogeneity $\left(\mathrm{I}^{2}=73.1 \%, P=0.001\right)$ using a random-effects model. The subgroup analysis, which divided the studies into a SPARCL1 overexpression group and a positive group, showed that overexpression of SPARCL1 was associated with a lower risk of distant metastasis (OR $=0.44$, 95\% CI: 0.23$0.85, \mathrm{Z}=2.46, P=0.014$ ). No significant difference was observed in the SPARCL1positive group $(\mathrm{OR}=0.84$, $P=0.739$ ) (Fig. 3). A sensitivity analysis was performed by sequential omission of individual studies using the random-effects model, and the result pattern was not obviously affected by any single study (Fig. 4). Further meta-regression showed that factors including race $(P=0.271)$, cancer type $(P=0.911)$ and sample size $(P=0.271)$ did not contribute to the heterogeneity. These results suggested that the overexpression of SPARCL1 might reduce the risk of distant metastasis of GI malignancies.

Six studies assessed the correlation between SPARCL1 expression and lymph node status. The

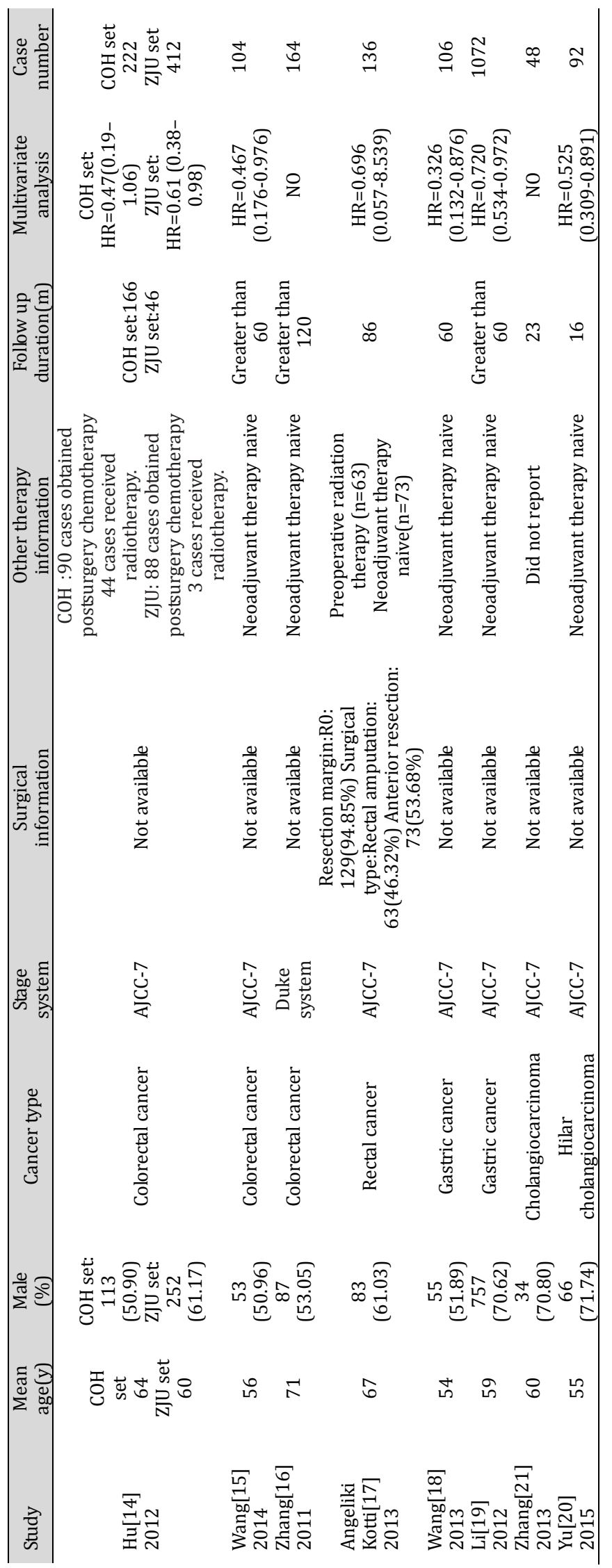


Fig. 2. Forest plots of studies evaluating hazard ratios of SPARCL1 expression and prognosis in GI malignancies.

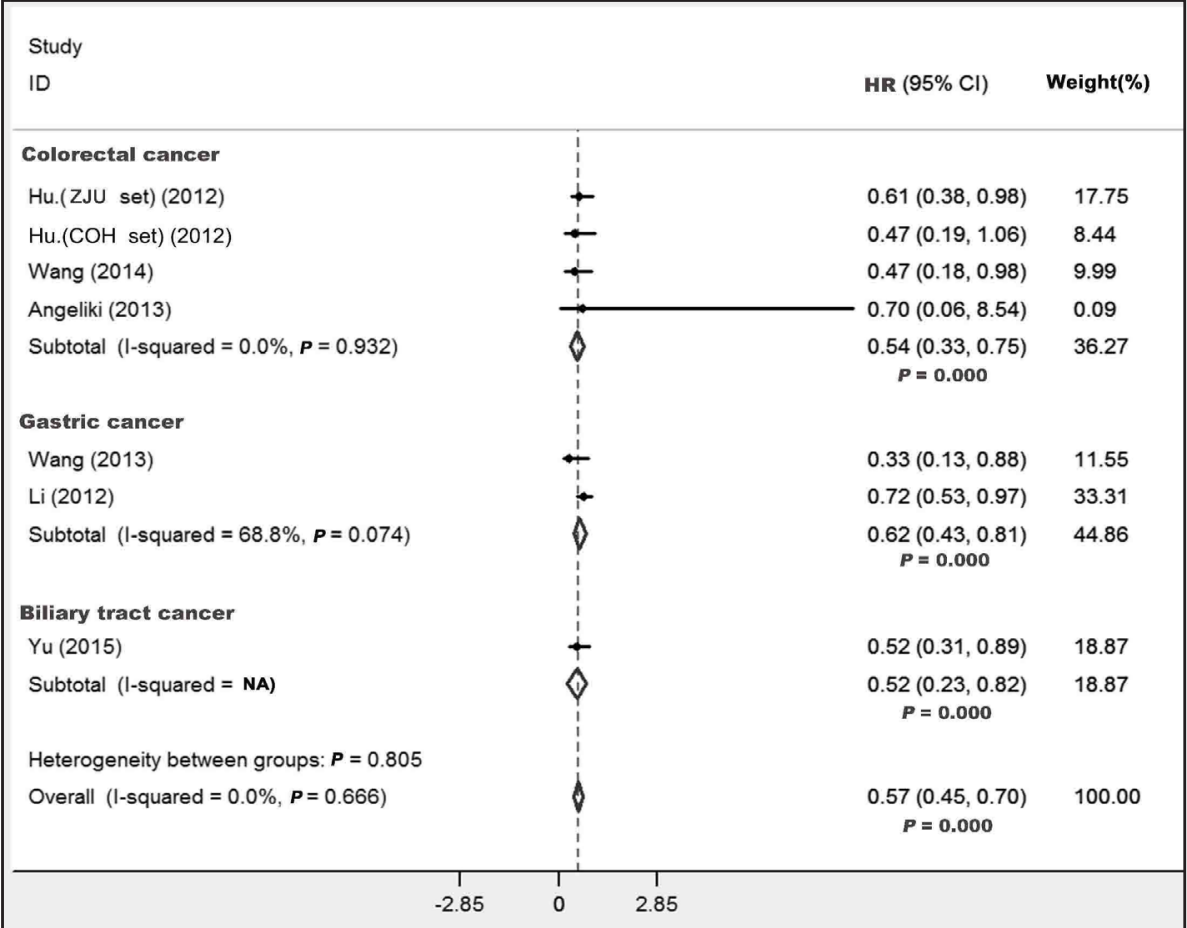

Fig. 3. The pooled OR of the association between SPARCL1 and GI malignancies' distant metastasis.

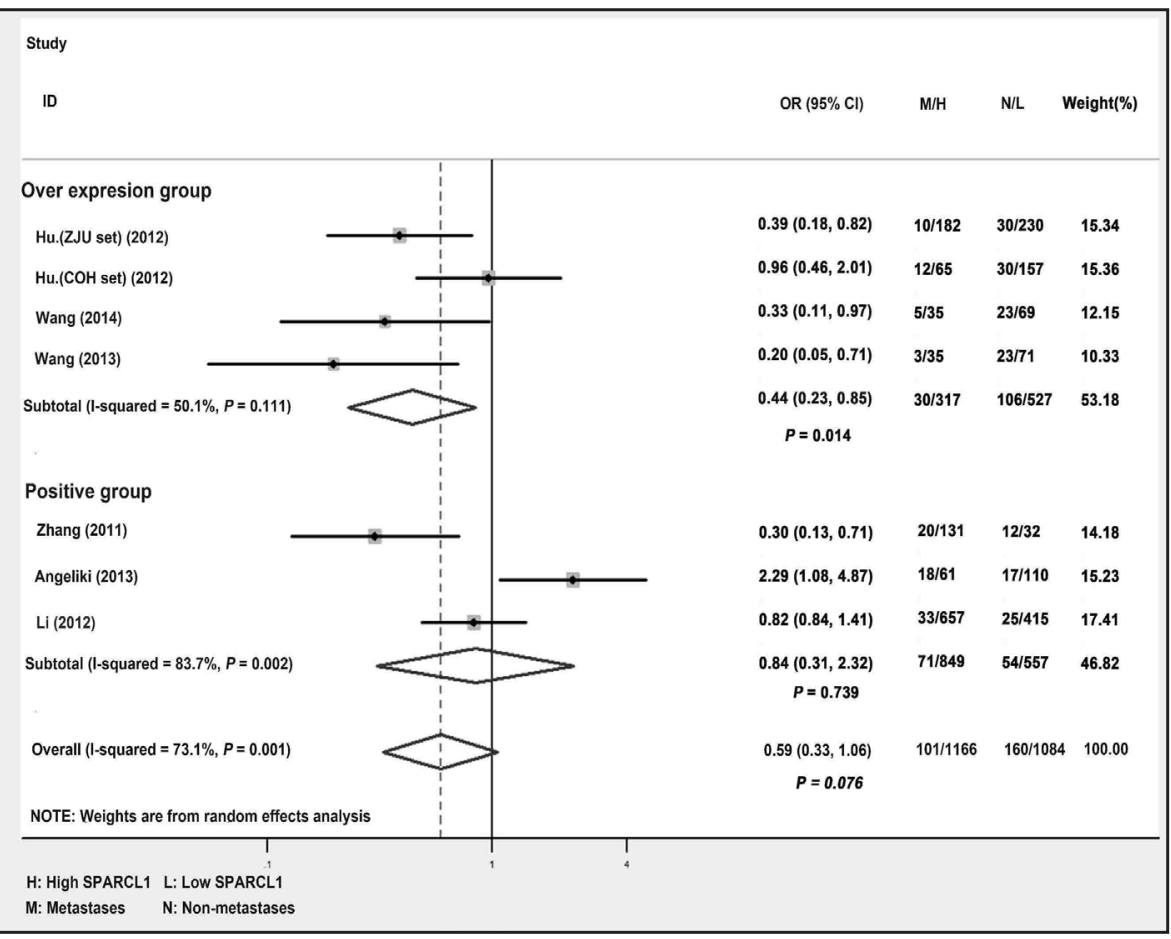

pooled OR was 0.56 (95\% CI: $0.39-0.81, \mathrm{Z}=3.12, P=0.002)$ with heterogeneity $\left(\mathrm{I}^{2}=57.4 \%\right.$, $P=0.029$ ) using a random-effects model (Fig. 5). The subgroup analysis revealed that colorectal cancer contributed to most of the heterogeneity $\left(I^{2}=68.1 \%, P=0.044\right)$, and expression of SPARCL1 might have no effect on lymph nodes status in colorectal cancer $(\mathrm{OR}=0.73, P=0.329)$. Expression of SPARCL1 reduced the risk of lymph node invasion in gastric cancer and biliary tract cancer. 
Fig. 4. Sensitivity analysis for meta-analysis of SPARCL1 expression.

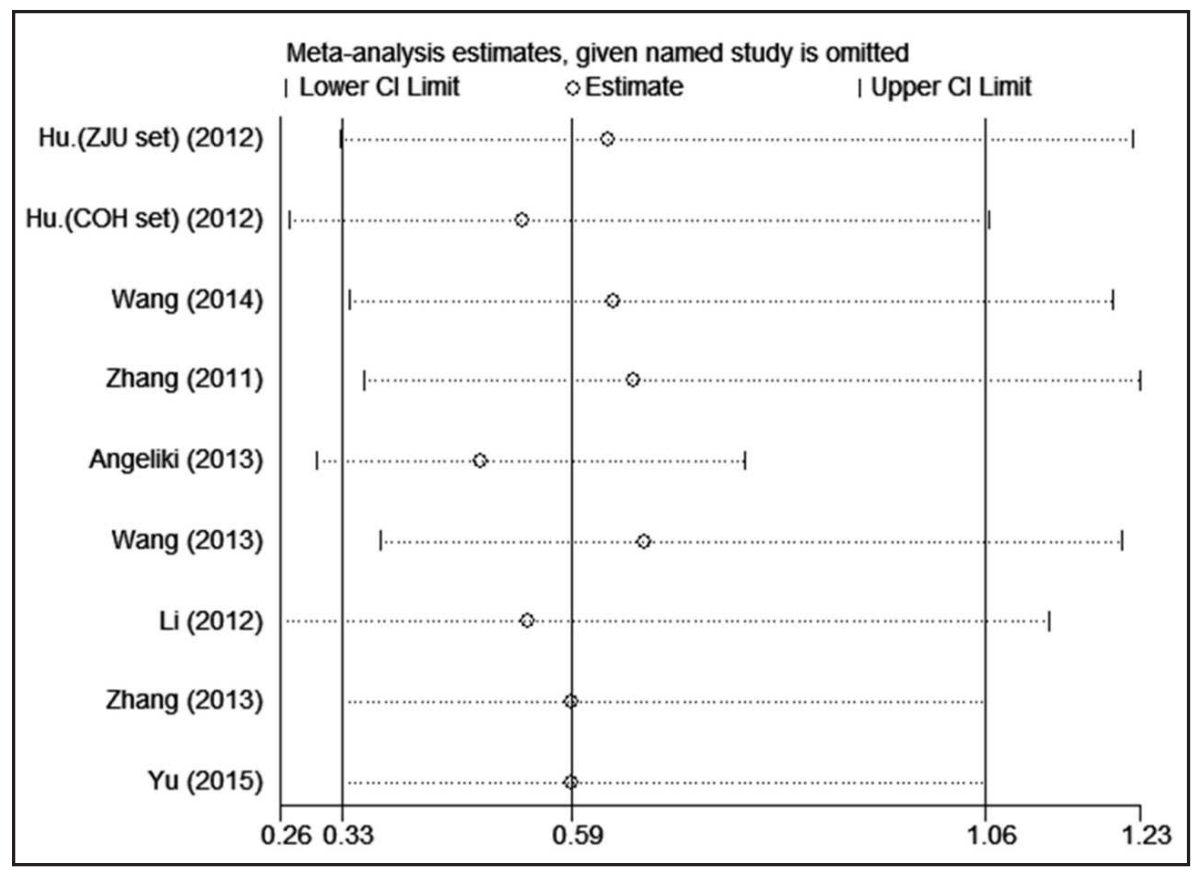

Fig. 5. The pooled OR of the association between SPARCL1 and GI malignancies' lymph nodes metastasis.

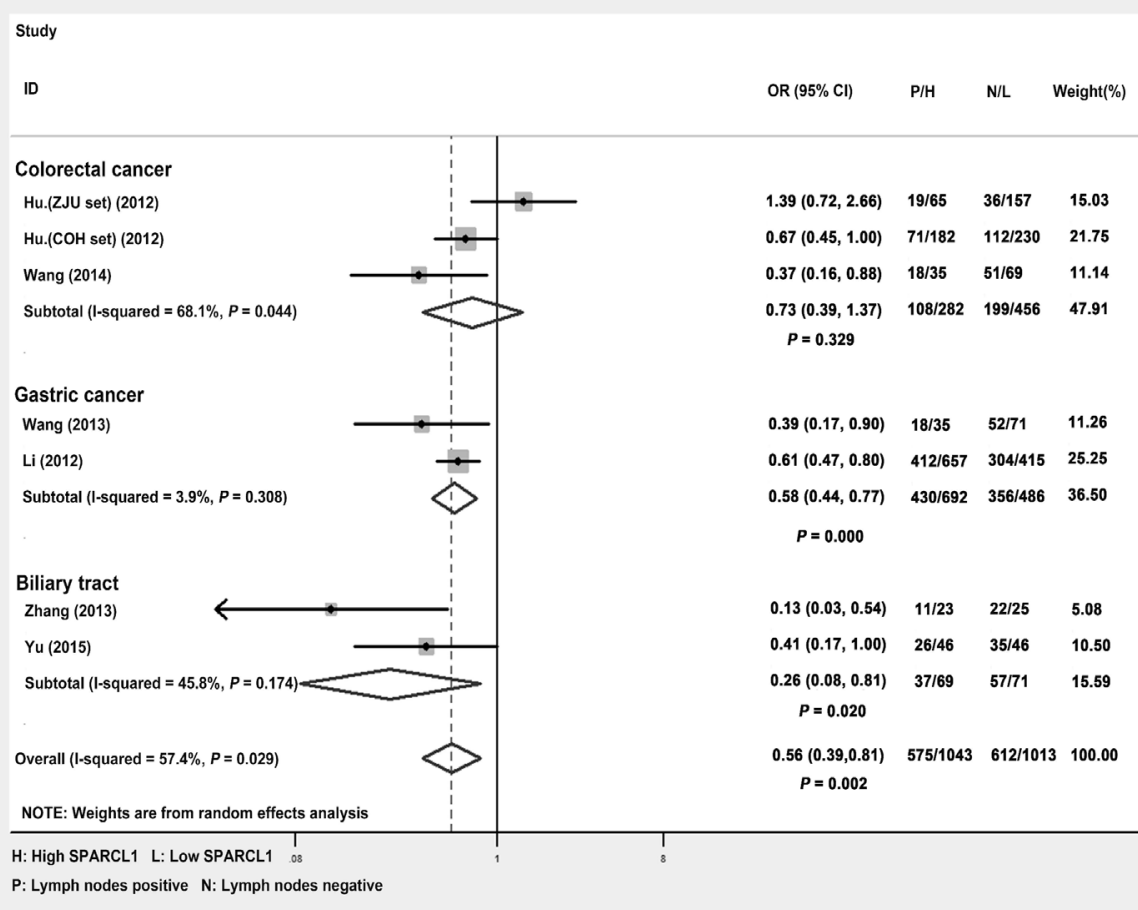

Seven studies assessed the association of the expression of SPARCL1 with tumor differentiation. The pooled OR was 2.21 (95\% CI: 1.82-2.69, $\mathrm{Z}=7.95, P=0.000$ ) with heterogeneity $\left(\mathrm{I}^{2}=25.9 \%, P=0.222\right)$ using a fixed-effects model. A subgroup analysis was performed, which revealed that the gastric cancer group $\left(\mathrm{I}^{2}=85.8 \%, P=0.008\right)$ was the main contributor to the heterogeneity. Expression of SPARCL1 indicated better differentiation in all cancer types (colorectal cancer: $\mathrm{OR}=2.05, P=0.000$; gastric cancer: $\mathrm{OR}=2.21, P=0.000$; biliary tract cancer: $\mathrm{OR}=3.21, P=0.006$ ) (Fig. 6). Therefore, we concluded that expression of SPARCL1 suggested better differentiation of GI malignancies. 


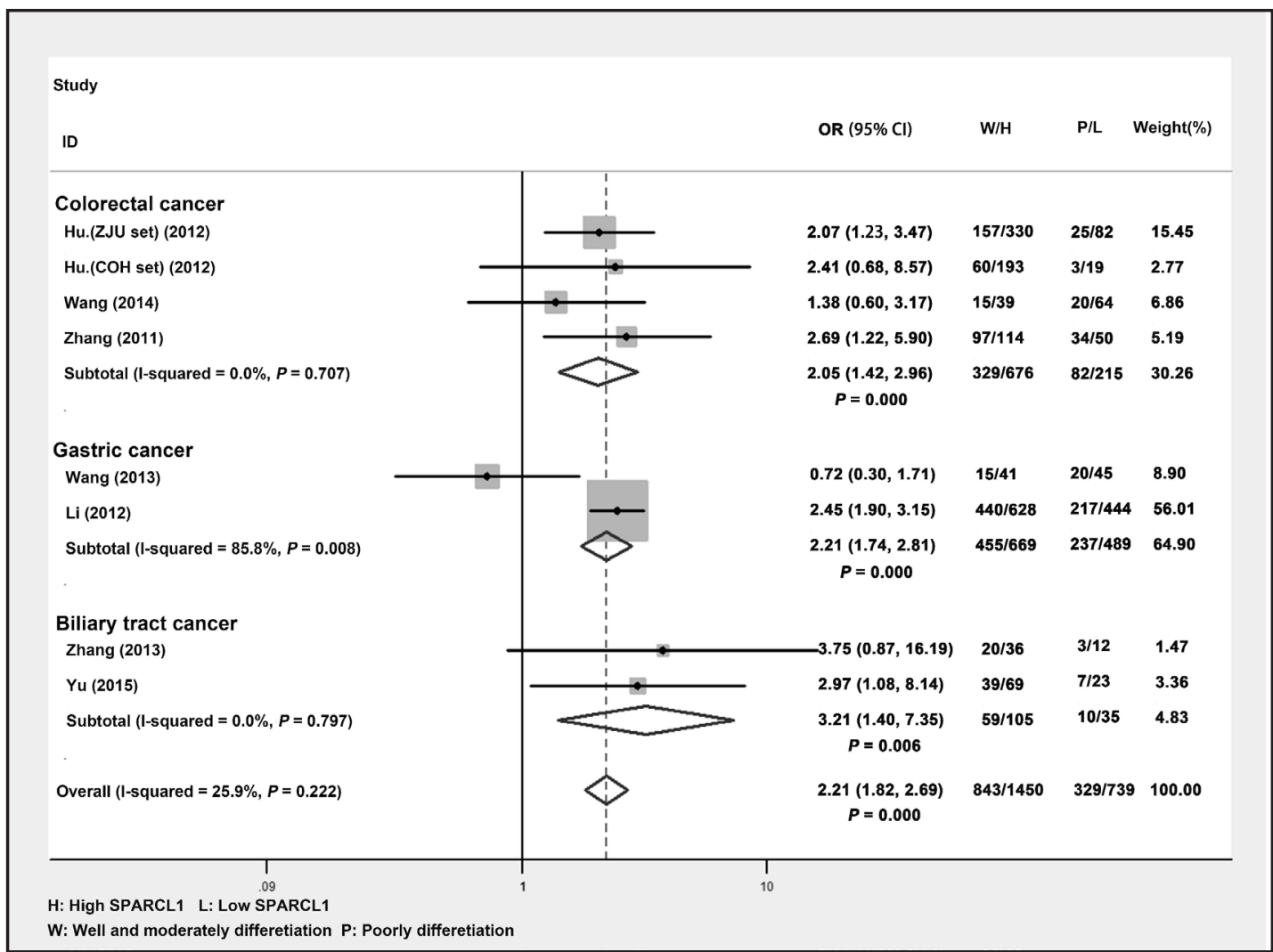

Fig. 6. The pooled OR of the association between SPARCL1 and GI malignancies' differentiation.

Fig. 7. Funnel plots of publication biases.

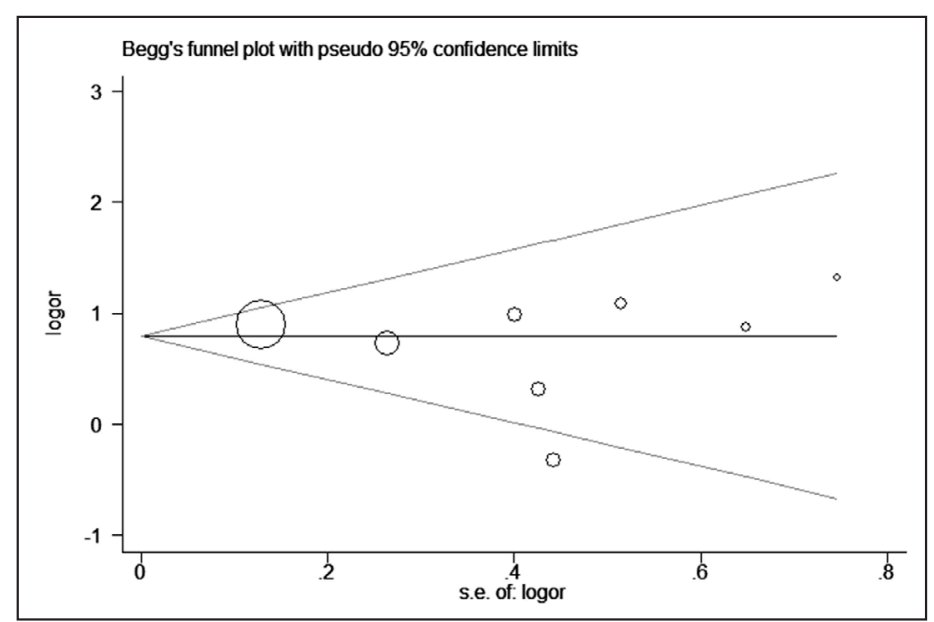

\section{Publication bias}

The publication bias of the included studies was evaluated using funnel plots and Begg's test, as shown in Fig. 7. Publication bias was not observed, and this conclusion was confirmed by Egger's test $(P>0.05)$. Hence, publication bias was not present in this meta-analysis, which indicates that our results are reliable. 


\section{Cellular Physiology Cell Physiol Biochem 2017;44:1485-1496

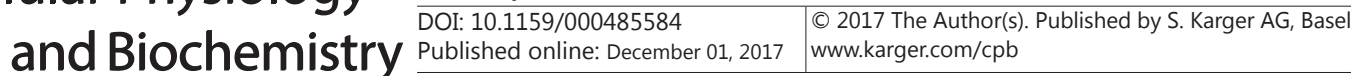 \\ Hanguang et al.: SPARCL1 as a Prognostic Marker for GI Malignances}

\section{Discussion}

Abnormal expression of the SPARCL1 gene and protein have been reported during the development and progression of many types of cancer, including non-small cell lung carcinomas [23], prostate carcinoma [24], colorectal carcinoma [14-17], gastric cancer [18, 19], pancreatic cancer [9], hepatocellular carcinoma [5], osteosarcoma [25] and gliomas [26]. The diverse expression pattern of SPARCL1 in different human malignancies suggests that it might play different roles in tumor biology based on the tumor type [2]. In the field of GI malignancies, most studies have indicated that down-regulation of the SPARCL1 protein leads to a poor prognosis in patients; however, the conclusions are controversial $[5,9,16$, 17]. Therefore, we performed this meta-analysis to explore the role of the SPARCL1 protein in GI malignancies. To the best of our knowledge, this is the first meta-analysis that was focused on the prognostic value of SPARCL1 protein expression in GI malignancies.

Our meta-analysis results showed that SPARCL1 could be a novel prognostic predictive factor for GI malignancies. Using a fixed-effects model, our analysis combined six published articles and revealed that the expression of SPARCL1 suggested a significantly better prognosis for GI malignancies (HR=0.572, $P=0.000$ ). We hypothesized that the expression of SPARCL1 affects the clinicopathological features of GI malignancies, which could explain why the expression of SPARCL1 results in a better prognosis. Therefore, we collected available data from eligible articles and further explored the relationship between the expression of SPARCL1 and the clinicopathological features of GI malignancies, including distant metastasis, lymph node status and tumor differentiation. The results showed that overexpression of SPARCL1 was associated with a reduction in distant metastasis (OR $=0.44$, $P=0.014$ ) and a lower risk of lymph node metastasis (OR=0.56, $P=0.002$ ).

As a secreted extracellular matrix glycoprotein, the adhesive functions of SPARCL1 on endothelial cells would likely contribute to the temporal regulation of the "angioendothelial" compartment of desmoplasia, with subsequent modulation of tumor cell invasion [27]. This hypothesis had been demonstrated by many researches. Hambrock et al. reported SPARCL1 in the extracellular matrix (ECM) tethers to the collagen ECM [28]. Hurley et al. and his colleagues demonstrate that SPARCL1 in the ECM limited cellular ability to form focal adhesions on the collagen matrix and thereby attenuated corresponding biophysical dynamics of the cytoskeletal framework, which have been shown to potentiate migration [29]. SPARCL1 mitigates mediators of focal adhesion activation such as RHO family members also has been proved [30,31]. Naschberger et al. reported that the heterogeneity of tumor microenvironments is regulated by SPARCL1, which promotes cell quiescence and vessel homeostasis and contributes to a favorable prognosis in colorectal carcinoma [32]. According to the results of our meta-analysis, the overexpression of SPARCL1 may reduce cancer metastasis (including lymph node and distant metastases) which contributes to survival benefits. Furthermore, some scientists have concluded that SPARCL1 gene plays a suppressor role in tumor metastasis in various cancers [24, 33, 34]. Our results also showed that the expression of SPARCL1 indicated better tumor differentiation (OR=2.21, $P=0.000$ ). The SPARCL1 gene is expressed during the embryonic development stage in many tissues and organs such as the heart, lungs, brain, bone, muscle, colon and lymphatic glands; however, it exhibits lower expression in the pancreas, spleen, thyroid gland and placental tissue [35, 36]. Therefore, SPARCL1 expression may play an important role throughout the growth and development of organs. Differentiation is a vital process during the growth and development of organs. Down-regulation of SPARCL1 may lead to poor differentiation, which may causes aggressive behavior of tumors and a poor prognosis for patients. This is consistent with our meta results that the expression of SPARCL1 indicated better tumor differentiation $(\mathrm{OR}=2.21$, $P=0.000$ ). These all above suggests that SPARCL1 restrict cellular invasion and regulate the tumor microenviroments. We guess this is the main anti-tumorigenic effect of SPARCL1. In addition, some of the evidence is correlative and by itself does not make a strong case for SPARCL1 as a regulator of tumor cell proliferation and angiogenesis [7, 36-38]. Our metaanalysis demonstrates that the expression of SPARCL1 may reduce the risk of lymph node 


\section{Cellular Physiology Cell Physiol Biochem 2017;44:1485-1496 \begin{tabular}{l|l|l} 
DOI: 10.1159/000485584 & O 2017 The Author(s). Published by S. Karger AG, Basel \\
www.karger.com/cpb
\end{tabular}}

Hanguang et al.: SPARCL1 as a Prognostic Marker for GI Malignances

metastasis and distant metastasis and promote tumor cell differentiation. We suspected that these functions would contribute to better survival of GI malignancy patients; however, the functions that play a leading role in the survival outcome depend on the specific cancer type. More research on the tumor formation mechanism is needed to confirm this conclusion.

As the first meta-analysis that is focused on the role of SPARCL1 in GI malignancies, there are some limitations in the included studies that need to be mentioned. First, the lack of a unified cut-off value of SPARCL1 expression resulted in the use of various judgement criteria in the eligible studies. The cut-off values may affect the efficiency of SPARCL1 as a prognostic biomarker. In our analysis, we divided studies into an overexpression group and a positive group for subgroup analysis, and the difference in results of the two groups is obvious. Identification of the most appropriate cut-off values for use of SPARCL1 as a prognostic predictive factor needs to be further explored. Second, the main ethnic populations investigated were Asian, while other ethnicities such as Africans were not included in the analysis. Third, the surgical information of patients which may affect patients' prognosis to some degree was unavailable [39-41]. Therefore, we advocate scientists and physicians to collect more clinical features such as surgical information and consider them into further analysis, which will make a stronger conclusion that SPARCL1 can be a prognosis factor.

\section{Conclusion}

Our study showed that the expression of SPARCL1 is associated with a better prognosis of GI malignancies. We propose that the expression of SPARCL1 could influence the clinicopathological features of GI malignancies, including tumor metastasis, lymph node status and tumor differentiation. The predominant clinicopathological features might be related to specific cancer types. We advocate that further large-scale studies are essential to confirm the prognostic predictive value of SPARCL1 which had better to consider more clinical feature of patients especially some factors may affect prognosis, and more fundamental experimental studies are needed to illustrate the mechanisms.

\section{Acknowledgements}

This study was supported by National Natural Science Foundation of China (81401935 \& 81300341); Zhejiang Provincial Natural Science Foundation of China (LQ13H160002); and National Human Genetic Resources Sharing Service Platform (2005DKA21300).

\section{Disclosure Statement}

The authors declare no conflict of interest.

\section{References}

1 Girard JP, Springer TA: Cloning from purified high endothelial venule cells of hevin, a close relative of the antiadhesive extracellular matrix protein SPARC. Immunity 1995;2:113-123.

-2 Gagliardi F, Narayanan A, Mortini P: SPARCL1 a novel player in cancer biology. Crit Rev Oncol Hematol 2017;109:63-68.

3 Chen W, Zheng R, Baade PD, Zhang S, Zeng H, Bray F, Jemal A, Yu XQ He J: Cancer statistics in China, 2015 CA Cancer J Clin 2016;66:115-132.

4 Pourhoseingholi MA, Vahedi M, Baghestani AR: Burden of gastrointestinal cancer in Asia; an overview. Gastroenterol Hepatol Bed Bench 2015;8:19-27. 


\section{Cellular Physiology Cell Physiol Biochem 2017;44:1485-1496 \begin{tabular}{l|l} 
and Biochemistry & DOI: 10.1159/000485584 \\
Published 2017 The Author(s). Published by S. Karger AG, Basel \\
www.karger.com/cpb
\end{tabular}

5 Lau CP, Poon RT, Cheung ST, Yu WC, Fan ST: SPARC and Hevin expression correlate with tumour angiogenesis in hepatocellular carcinoma. J Pathol 2006;210:459-468.

6 Jakharia A, Borkakoty B, Singh S: Expression of SPARC like protein 1 (SPARCL1), extracellular matrixassociated protein is down regulated in gastric adenocarcinoma. J Gastrointest Oncol 2016;7:278-283.

7 Claeskens A, Ongenae N, Neefs JM, Cheyns P, Kaijen P, Cools M, Kutoh E: Hevin is down-regulated in many cancers and is a negative regulator of cell growth and proliferation. Br J Cancer 2000;82:1123-1130.

-8 Girard JP, Springer TA: Modulation of endothelial cell adhesion by hevin, an acidic protein associated with high endothelial venules. J Biol Chem 1996;271:4511-4517.

-9 Esposito I, Kayed H, Keleg S, Giese T, Sage EH, Schirmacher P, Friess H, Kleeff J: Tumor-suppressor function of SPARC-like protein 1/Hevin in pancreatic cancer. Neoplasia 2007;9:8-17.

10 Parmar MK, Torri V, Stewart L: Extracting summary statistics to perform meta-analyses of the published literature for survival endpoints. Stat Med 1998;17:2815-2834.

11 DerSimonian R, Laird N: Meta-analysis in clinical trials. Control Clin Trials 1986;7:177-188.

12 Mantel N, Haenszel W: Statistical aspects of the analysis of data from retrospective studies of disease. J Natl Cancer Inst 1959;22:719-748.

13 Higgins JP, Thompson SG, Deeks JJ, Altman DG: Measuring inconsistency in meta-analyses. BMJ 2003;327:557-560.

14 Hu H, Zhang H, Ge W, Liu X, Loera S, Chu P, Chen H, Peng J, Zhou L, Yu S, Yuan Y, Zhang S, Lai L, Yen Y, Zheng $\mathrm{S}$ : Secreted protein acidic and rich in cysteines-like 1 suppresses aggressiveness and predicts better survival in colorectal cancers. Clin Cancer Res 2012;18:5438-5448.

15 Wang X, Zhou Q, Feng Q Zhang B, Li X, Tao D, Hu J, Gong J: The relationship between clinical significance of colorectal cancer and the expression of SPARCL1. J Practic Med 2014;14:2242-2245.

-16 Zhang H, Widegren E, Wang DW, Sun XF: SPARCL1: a potential molecule associated with tumor diagnosis, progression and prognosis of colorectal cancer. Tumour Biol 2011;32:1225-1231.

17 Kotti A, Holmqvist A, Albertsson M, Sun XF: SPARCL1 expression increases with preoperative radiation therapy and predicts better survival in rectal cancer patients. Int J Radiat Oncol Biol Phys 2014;88:11961202.

18 Wang X, Wang H, Zhang T, Han L, Yang Y, Yuan J: The relationship between clinical significance of gastric cancer and the expression of SPARCL1. Chin J Exp Surg 2013;30:236-238.

19 Li P, Qian J, Yu G, Chen Y, Liu K, Li J, Wang J: Down-regulated SPARCL1 is associated with clinical significance in human gastric cancer. J Surg Oncol 2012;105:31-37.

20 Yu Y, Chen Y, Ma J, Yu X, Yu G, Li Z: SPARCL1 is a novel predictor of tumor recurrence and survival in hilar cholangiocarcinoma. Tumour Biol 2016;37:4159-4167.

21 Zhang H, Shi W, Song N, Wang X: The relationship between clinical significance of billiary tract cancer and the expression of SPARCL1. China Oncology 2013;2:126-129.

-22 Stang A: Critical evaluation of the Newcastle-Ottawa scale for the assessment of the quality of nonrandomized studies in meta-analyses. Eur J Epidemiol 2010;25:603-605.

23 Bendik I, Schraml P, Ludwig CU; Characterization of MAST9/Hevin, a SPARC-like protein, that is downregulated in non-small cell lung cancer.Cancer Res 1998;58:626-629 .

-24 Hurley PJ, Marchionni L, Simons BW, Ross AE, Peskoe SB, Miller RM, Erho N, Vergara IA, Ghadessi M, Huang Z, Gurel B, Park BH, Davicioni E, Jenkins RB, Platz EA, Berman DM, Schaeffer EM: Secreted protein, acidic and rich in cysteine-like 1 (SPARCL1) is down regulated in aggressive prostate cancers and is prognostic for poor clinical outcome. Proc Natl Acad Sci U S A 2012;109:14977-14982.

-25 Sullivan MM, Sage EH: Hevin/SC1, a matricellular glycoprotein and potential tumor-suppressor of the SPARC/BM-40/Osteonectin family. Int J Biochem Cell Biol 2004;36:991-996.

-26 Turtoi A, Musmeci D, Naccarato AG, Scatena C, Ortenzi V, Kiss R, Murtas D, Patsos G, Mazzucchelli G, De Pauw E, Bevilacqua G, Castronovo V: Sparc-like protein 1 is a new marker of human glioma progression. J Proteome Res 2012;11:5011-5021.

27 Framson PE, Sage EH: SPARC and tumor growth: where the seed meets the soil. J Cell Biochem 2004;92:679-690.

28 Hambrock HO, Nitsche DP, Hansen U, Bruckner P, Paulsson M, Maurer P, Hartmann U: SC1/hevin. An extracellular calcium-modulated protein that binds collagen I. J Biol Chem 2003;278:11351-8 . 


\section{Cellular Physiology Cell Physiol Biochem 2017;44:1485-1496

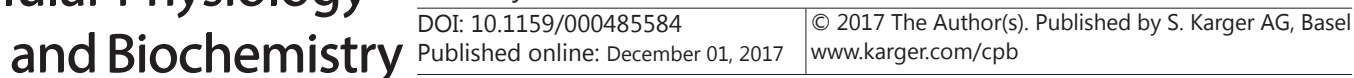

Hanguang et al.: SPARCL1 as a Prognostic Marker for GI Malignances

29 Hurley PJ, Hughes RM, Simons BW, Huang J, Miller RM, Shinder B, Haffner MC, Esopi D, Kimura Y, Jabbari J, Ross AE, Erho N, Vergara IA, Faraj SF, Davicioni E, Netto GJ, Yegnasubramanian S, An SS, Schaeffer EM: Androgen-Regulated SPARCL1 in the Tumor Microenvironment Inhibits Metastatic Progression. Cancer Res 2015;75:4322-4334.

30 Sullivan MM, Puolakkainen PA, Barker TH, Funk SE, Sage EH: Altered tissue repair in hevin-null mice: inhibition of fibroblast migration by a matricellular SPARC homolog. Wound Repair Regen 2008;16:310319.

31 Hurley PJ, Marchionni L, Simons BW, Ross AE, Peskoe SB, Miller RM, Erho N, Vergara IA, Ghadessi M, Huang Z, Gurel B, Park BH, Davicioni E, Jenkins RB, Platz EA, Berman DM, Schaeffer EM: Secreted protein, acidic and rich in cysteine-like 1 (SPARCL1) is down regulated in aggressive prostate cancers and is prognostic for poor clinical outcome. Proc Natl Acad Sci U S A 2012;109:14977-14982.

32 Naschberger E, Liebl A, Schellerer VS, Schütz M, Britzen-Laurent N, Kölbel P, Schaal U, Haep L, Regensburger D, Wittmann T, Klein-Hitpass L, Rau TT, Dietel B, Méniel VS, Clarke AR, Merkel S, Croner RS, Hohenberger W, Stürzl M: Matricellular protein SPARCL1 regulates tumor microenvironment-dependent endothelial cell heterogeneity in colorectal carcinoma. J Clin Invest 2016;126:4187-4204.

-33 Xiang Y, Qiu Q Jiang M, Jin R, Lehmann BD, Strand DW, Jovanovic B, DeGraff DJ, Zheng Y, Yousif DA, Simmons CQ Case TC, Yi J, Cates JM, Virostko J, He X, Jin X, Hayward SW, Matusik RJ, George AL, Yi Y: SPARCL1 suppresses metastasis in prostate cancer. Mol Oncol 2013;7:1019-1030.

-34 Cao F, Wang K, Zhu R, Hu YW, Fang WZ, Ding HZ: Clinicopathological significance of reduced SPARCL1 expression in human breast cancer. Asian Pac J Cancer Prev 2013;14:195-200.

-35 Hambrock HO, Nitsche DP, Hansen U, Bruckner P, Paulsson M, Maurer P, Hartmann U: SC1/hevin. An extracellular calcium-modulated protein that binds collagen I. J Biol Chem 2003;278:11351-11358.

-36 Mencalha AL,Levinsphul A,Deterling LC,Pizzatti L,Abdelhay E.SPARC-like1 mRNA is over expessed in human uterine leiomyoma [J].Molecular medicine reports. 2008 Jul-Aug;1(4):571-4.

-37 Fidler IJ: The pathogenesis of cancer metastasis: the 'seed and soil' hypothesis revisited. Nat Rev Cancer 2003;3:453-458.

38 Zaravinos A, Lambrou GI, Boulalas I, Delakas D, Spandidos DA: Identification of common differentially expressed genes in urinary bladder cancer. PLoS One 2011;6:e18135.

-39 Gasparini G, Harris AL: Clinical importance of the determination of tumor angiogenesis in breast carcinoma: much more than a new prognostic tool. J Clin Oncol 1995;13:765-782.

40 West NP, Morris EJ, Rotimi O, Cairns A, Finan PJ, Quirke P: Pathology grading of colon cancer surgical resection and its association with survival: a retrospective observational study. Lancet Oncol 2008;9:857865.

41 Madoff RD: Defining quality in colon cancer surgery. J Clin Oncol 2012;30:1738-1740. 